\title{
Electrical decoupling effect on intermediate band Ti-implanted silicon layers
}

\author{
D Pastor , J Olea , A del Prado , E García-Hemme ， \\ R García-Hernansanz , I Mártil and G González-Díaz
}

\begin{abstract}
We investigated the electrical transport properties of ultraheavily Ti-implanted silicon layers subsequently pulsed laser melted (PLM). After PLM, the samples exhibit anomalous electrical behaviour in sheet resistance and Hall mobility measurements, which is associated with the formation of an intermediate band (IB) in the implanted layer. An analytical model that assumes IB formation and a current limitation effect between the implanted layer and the substrate was developed to analyse this anomalous behaviour. This model also describes the behaviour of the function $V / \Delta V$ and the electrical function $F$ that can be extracted from the electrical measurements in the bilayer. After chemical etching of the implanted layer, the anomalous electrical behaviour observed in sheet resistance and Hall mobility measurements vanishes, recovering the unimplanted Si behaviour, in agreement with the analytical model. The behaviour of $V / \Delta V$ and the electrical function $F$ can also be successfully described in terms of the analytical model in the bilayer structure with the implanted layer entirely stripped.
\end{abstract}

(Some figures may appear in colour only in the online journal)

\section{Introduction}

The intermediate band (IB) solar cell concept has emerged as a promising candidate for high-efficiency solar cells in the third generation of photovoltaics [1]. An IB material is a semiconductor that presents a third band of allowed states between the conventional conduction band (CB) and valence band (VB). The IB permits sub-bandgap photon absorption, which pumps electrons from the VB to the $\mathrm{CB}$ using the IB as an intermediate step, and can overcome the ShockleyQueisser themodynamical efficiency limit for single junction solar cells [2]. Strong evidence of IB material formation and IB solar cell operation has been observed recently in quantum dot structures [3] and dilute compound alloys [4]. Another proposed approach to obtaining the IB materials is the introduction of a concentration of deep impurity levels above the Mott transition [5,6]. These impurity concentrations are usually well above the solid solubility limit and we need to use two non-equilibrium techniques to introduce the high concentration required such as ion implantation followed by a pulsed laser melting (PLM) process to recover the lattice damage produced during ion implantation. These techniques have been extensively used to obtain supersaturated materials $[7,8]$.

In order to elucidate IB formation in these supersaturated Si materials, we analysed the electrical transport properties of ultraheavily Ti-implanted Si samples. These bilayers showed an unexpected rectifying electrical behaviour, which we have attributed recently to an electrical decoupling effect, as a result of the IB formation in the Ti-supersaturated Si region $[9,10]$. We proposed an analytical two-layer model to explain the electrical transport properties of Ti-implanted $\mathrm{Si} / \mathrm{Si}$ substrate bilayers in terms of the IB formation in the Ti-supersaturated Si region [11]. Electrical transport 
measurements for different Ti implanted doses show that this anomalous rectifying electrical behaviour is observed only for samples with Ti concentrations well above the Mott transition [12]. For these concentrations, the measurements performed at low temperatures reveal that the Ti-supersaturated Si material presents metallic behaviour, which is consistent with a semifilled IB formation in the supersaturated Si material [13]. However, the underlying mechanism responsible for the electrical decoupling requires a deeper knowledge.

In this paper, we present the electrical transport properties of Ti-supersaturated silicon formed on top of a Si substrate to study this current limitation effect at the junction of the bilayer, which is associated with the IB formation and is responsible for the observed electrical decoupling behaviour. To validate the analytical model and probe that the anomalous electrical behaviour is uniquely produced due to the current limitation effect at the junction, which in turn is due to the IB formation in the Ti-supersaturated layer, we analyse the electrical behaviour of the bilayer before and after a chemical etching process that completely strips the implanted layer, but preserves the metal contacts in the corners of the samples, and the bilayer structure under these contacts. The electrical transport properties of this new structure and the possible current limitation effect may be consistently explained in terms of the analytical two-layer model cited above. After the corresponding simplifications in the analytical model to take into account the suppression of conductivity along the Ti-implanted layer, we would be able to predict the experimental behaviour in the new structure. These results could open new routes to characterize electrically and evidence IB material formation.

\section{Experimental}

Single crystal n-type Si (1 11 1) samples of thickness $300 \mu \mathrm{m}$ $\left(\rho=200 \Omega \mathrm{cm} ; \mu=1500 \mathrm{~cm}^{2} \mathrm{~V}^{-1} \mathrm{~s}^{-1} ; n=2.2 \times 10^{13} \mathrm{~cm}^{-3}\right.$ at RT) and area $1 \times 1 \mathrm{~cm}^{2}$ were implanted in an IBS refurbished VARIAN CF3000 ion implanter at $33 \mathrm{keV}$ with ${ }^{48} \mathrm{Ti}^{+}$with a dose of $10^{15} \mathrm{~cm}^{-2}$. We performed the implantations using a $7^{\circ}$ tilt angle to minimize channeling effects. After implantation, all the samples were PLM processed at $0.8 \mathrm{~J} \mathrm{~cm}^{-2}$ with a single $20 \mathrm{~ns}$ pulse using a $\mathrm{KrF}$ excimer laser $(248 \mathrm{~nm})$ at IPG Photonics (New Hampshire, USA).

The structural characterization of the Ti-supersaturated Si layers obtained with the same conditions analysed in this work reveals good crystal quality after the PLM process [14]. From the grazing incidence x-ray diffraction (GIXRD) patterns, transmission electron microscopy (TEM) and high-resolution transmission electron microscopy (HRTEM) images, electron diffraction (ED) patterns and Raman spectra no proofs of Ti-Si phases, precipitates or Ti clustering are detected $[9,15,16]$.

After the PLM process, $200 \mathrm{~nm}$ thick aluminum contacts were deposited on the corners of the sample (triangles of $1.5 \mathrm{~mm}$ side), on the implanted layer side, by means of electron beam evaporation. We electrically characterized the Si-implanted samples performing sheet resistance and Hall effect measurements with the van der Pauw configuration at variable temperature (90-300 K) using a Keithley SCS 4200 model with four source and measure units. We measured

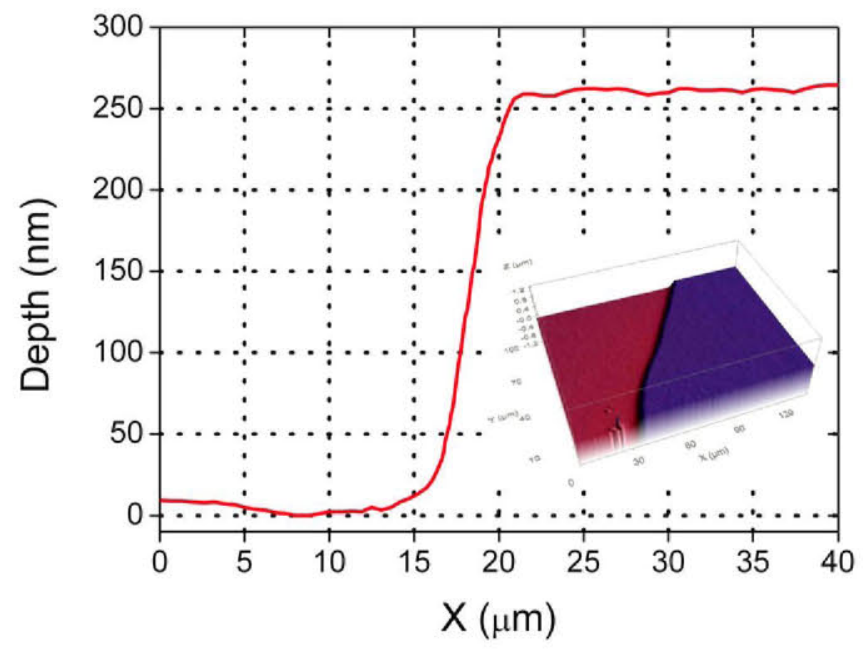

Figure 1. Depth profile of the edge in the etched region obtained with an optical profilometer. Inset: view of the topographical surface in the etched region.

the sheet resistance using the van der Pauw set-up. We measured the four possible van der Pauw rotations and we also changed the polarity of the measurement current source, by averaging the eight possible configurations. In all these configurations the current was injected between two adjacent contacts, measuring the voltage between the opposite contacts of the set-up. The Hall effect measurements were also performed using the same van der Pauw set-up. We measured the four possible van der Pauw rotations changing the magnetic field polarity, by averaging the eight possible configurations. In all these configurations the current was injected between two non-contiguous contacts, measuring the Hall voltage between the other two contacts. All these configurations in the sheet resistance and Hall effect measurements were employed to avoid any possibility of having spurious thermogalvanomagnetic effects.

In all the van der Pauw results presented in this work we applied the geometric factor [17]. After electrical characterization, the Ti-implanted layer of some of the samples was chemically etched preserving $\mathrm{Al}$ contacts with a photoresist mask. Wet chemical etching was performed using a $\mathrm{HNO}_{3}$-rich $\mathrm{HF}$ (2-98\%) mixture for $10 \mathrm{~s}$ [18]. After chemical etching, the photoresist was removed with acetone. The surface topography was investigated using a Sensofar confocal imaging profilometer, with a depth resolution better than $5 \mathrm{~nm}$, to determine the etching depth. The electrical measurements were repeated after removing the implanted layer.

\section{Results and discussion}

Figure 1 displays the depth profile of the edge in the etched region obtained with an optical profilometer. This profile reveals an edge of about $250 \mathrm{~nm}$, which is deeper than the Ti-implanted region of about $50 \mathrm{~nm}$. Details of the Ti-implanted profile can be found in [12]. The surface topography of the edge of an area of the etched region can be observed in the inset of figure 1 . 


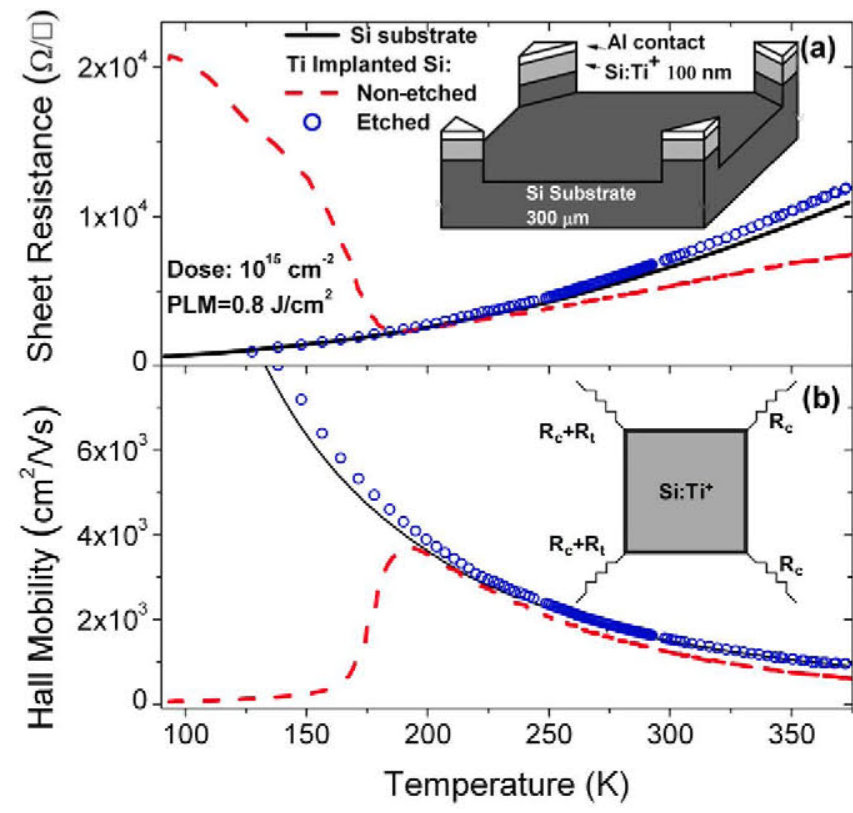

Figure 2. Sheet resistance $(a)$ and Hall Mobility $(b)$ of the etched and non-etched Ti-implanted silicon samples with a dose of $10^{15}$, PLM at $0.8 \mathrm{~J} \mathrm{~cm}^{-2}$. Measurements of the unimplanted Si substrate are also included. Insets $(a)$ : schematic view of the etched sample; (b) diagram of van der Pauw measurement where $R_{\mathrm{c}}$ is the contact resistance and $R_{\mathrm{t}}=1 / G_{\mathrm{t}}$ is the transversal resistance.

Figure 2(a) shows the sheet resistance in the 90-370 K temperature range of a Ti-implanted Si sample with a dose of $10^{15} \mathrm{~cm}^{-2}$ after the PLM process at $0.8 \mathrm{~J} \mathrm{~cm}^{-2}$ and the sheet resistance of the same sample after chemical etching of the Ti-implanted layer. The schematic view of the Ti-implanted Si sample after chemical etching is shown in the inset of figure 2(a). The sheet resistance of an unimplanted $\mathrm{Si}$ substrate is also shown for comparative purposes. As can be observed, the sheet resistance of the $\mathrm{Si}$ substrate decreases as the temperature is decreased due to a reduction in the scattering with the optical phonons of the lattice [19]. The sheet resistance of the implanted sample before etching presents anomalous behaviour at low temperatures that has been described previously [11]. Since the sheet resistance of a bilayer cannot be higher than the sheet resistance of one of the layers, we can conclude that an electrical decoupling effect related to a current limitation at the junction of the bilayer takes place. This would indicate that the substrate begins to disappear in the electrical measurements, for temperatures below $200 \mathrm{~K}$, and eventually we will measure only the sheet resistance of the implanted layer. This behaviour has been satisfactorily explained and described elsewhere by means of an analytical model in terms of the IB formation in the implanted layer [11]. In this analytical model the sheet resistance of the bilayer is described as

$$
R_{\text {sheet }}=\frac{G_{p 1}+G_{p 2} F^{2}}{\alpha\left(G_{p 1}+G_{p 2} F\right)^{2}}
$$

where $G_{p 1}$ is the conductance in the Ti-implanted layer measured between two contiguous contacts, $G_{p 2}$ is the conductance in the unimplanted $\mathrm{Si}$ substrate layer between two of the contacts, $\alpha$ is the relation between the sheet resistance $R_{s}$ of a layer and the resistance seen between two of the contacts [11], which depends on the geometrical relation between the electrode size and the sample size. For the parameters of the samples used in this work $\alpha \sim 2 . F$ is a function that takes into account the electrical decoupling effect observed in figure 2(a). This function is defined as $F=G_{\mathrm{t}} /\left(G_{\mathrm{t}}+G_{p 2}\right)$; where $G_{\mathrm{t}}$ is the transversal conductance under the contacts between the implanted layer and the Si substrate when a reverse bias voltage is applied. This conductance depends on the IB energy level position $\left(E_{\mathrm{IB}}\right)$ and is formulated as $G_{\mathrm{t}}=1 / R_{\mathrm{t}}=A^{-1} \exp \left(E_{\mathrm{IB}}-E_{\mathrm{c}} / k T\right), A$ being temperature independent [11].

In equation (1), $R_{\text {sheet }}$ tends to $1 /\left(\alpha G_{p 1}\right)=R_{\mathrm{s} 1}$ when $G_{\mathrm{t}} \ll G_{p 2}$ and hence $F$ tend to zero (which means fully decoupled) and $R_{\text {sheet }}$ tends to $1 /\left[\alpha\left(G_{p 1}+G_{p 2}\right)\right]$ when $G_{\mathrm{t}} \gg G_{p 2}$ and hence $F$ tends to 1 (fully coupled).

In the case in which the Ti-implanted layer is etched, the electrical conduction in the implanted layer, $G_{p 1}$ vanishes. Then, if $G_{p 1}=0$ in equation (1), we obtain

$$
R_{\text {sheet }}=\frac{1}{\alpha G_{p 2}}=R_{s 2}
$$

which is the sheet resistance of the unimplanted Si substrate.

However, the area under the $\mathrm{Al}$ contacts is not etched and the Ti-implanted Si layer is still present. Then, Ti-implanted $\mathrm{Si} / \mathrm{Si}$ substrate junctions are present and the transversal conductance $G_{\mathrm{t}}$ due to the presence of IB should exist in these regions. Nevertheless, due to the lack of current paths through the implanted layer, the Ti-implanted regions under the contacts are unconnected, and the resistance associated with these bilayer junctions behaves like a resistance in series with the metal contact. Since the van der Pauw method is not sensitive to serial resistances, these resistances are not observed in the experimental measurements of sheet resistance and Hall mobility. The inset of figure $2(b)$ displays the van der Pauw diagram of the etched sample.

As shown in figure $2(a)$, the sample after etching the implanted layer exhibits the same behaviour as the unimplanted Si substrate, as can be expected if the electrical conduction in the implanted layer disappears, in agreement with equation (2). Since the metallic contacts were exactly the same before and after chemical etching, the anomalous rectifying electrical behaviour observed in sheet resistance before the etching process cannot be attributed to temperaturedependent rectifying Schottky behaviour of the electrical contacts. Therefore, this rectifying electrical behaviour could be exclusively attributed to the decoupling effect in the bilayer junction due to a current limitation effect associated with IB formation [11]. Figure 2(b) displays the Hall mobility (n-type) measurements in the 90-370 K temperature range of the same samples of figure 2(a). The Hall mobility behaviour of the Ti-implanted sample before the etching process shows a strong reduction around $200 \mathrm{~K}$, in opposition to the behaviour expected for an unimplanted $\mathrm{Si}$ substrate, which tends to increase as the temperature is decreased due to a reduction in the lattice phonon scattering. These electrical measurements have been previously reported [11]. 


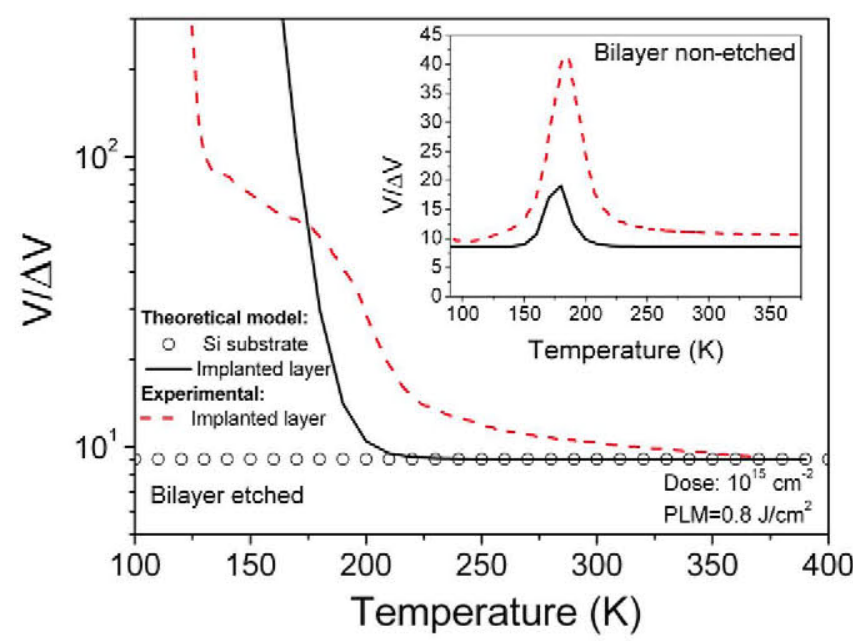

Figure 3. $V / \Delta V$ relation of the etched sample and the fit to the two-layer model of the Ti-implanted $\mathrm{Si}$ sample. Inset: $V / \Delta V$ relation of the non-etched sample and the fit to the two-layer model of the Ti-implanted Si sample.

Moreover, the Hall mobility of the Ti-supersaturated Si sample before etching is well explained again taking into account the electrical decoupling in the bilayer due to IB formation in the implanted layer [10]. The analytical model formulates the Hall mobility in the bilayer as [11]

$$
\mu_{\mathrm{eff}}=\frac{-\mu_{n 1} G_{\mathrm{pe} 1}+\mu_{h 1} G_{\mathrm{ph} 1}-\mu_{n 2} G_{p 2} F^{2}}{G_{\mathrm{pe} 1}+G_{\mathrm{ph} 1}+G_{p 2} F^{2}}
$$

where in this case $G_{\mathrm{pe} 1}$ and $\mu_{n 1}$ are, respectively, the conductance between two contiguous contacts and the mobility associated with the CB of the Ti-implanted layer, $G_{\mathrm{ph} 1}$ and $\mu_{h 1}$ are, respectively, the conductance and the mobility of the IB of the Ti-implanted layer, and $G_{p 2}$ and $\mu_{n 2}$ are, respectively, the conductance and the mobility in the $\mathrm{CB}$ of the unimplanted $\mathrm{Si}$ substrate. If the implanted layer is etched, conduction by this layer disappears and in equation (3), $G_{\mathrm{pe} 1}, \mu_{n 1}, G_{\mathrm{ph} 1}$ and $\mu_{h 1}$ are zero, obtaining finally, $\mu_{\text {eff }}=\mu_{n 2}$, which is the mobility of the electrons in the $\mathrm{CB}$ of the unimplanted Si substrate. In figure $2(b)$, the Hall mobility measurement of the chemically etched sample exhibits again the Si substrate behaviour as was expected and in agreement with the analytical model [11].

The analytical two-layer model that analyses the sheet resistance also considers the $V / \Delta V$ factor in the van der Pauw set-up, defining $V$ as the voltage developed at the current source when the current is injected into the electrodes and $\Delta V$ as the voltage difference generated in the opposite corners of the van der Pauw set-up.

In figure 3 we show the $V / \Delta V$ relation as a function of the temperature for the Ti-implanted sample after the chemical etching process. Theoretical behaviour of a $\mathrm{Si}$ substrate is also included. In the inset of figure 3 the $V / \Delta V$ factor is shown as a function of the temperature for a Ti-supersaturated $\mathrm{Si}$ sample before the chemical etching. If the contacts do not limit the current, the $V / \Delta V$ relation behaviour is expressed in the bilayer analytical model as

$$
\frac{V}{\Delta V}=\frac{\alpha \pi}{\ln (2)} \frac{G_{p 1}+G_{p 2} F}{G_{p 1}+G_{p 2} F^{2}}
$$

As can be appreciated from this expression, $V / \Delta V$ tends to $\alpha \pi / \ln 2$ when $F$ tends to 0 or $1\left(G_{\mathrm{t}} \ll G_{p 2}\right.$ or $G_{\mathrm{t}} \gg G_{p 2}$, respectively). This latter case also corresponds to a silicon single layer with ohmic contacts. In the inset, the theoretical prediction of the analytical model is compared with the experimental results obtained from [11]. The experimental results obtained from this sample are in agreement with the theoretical predictions of the analytical model [11].

Once the Ti-implanted layer is etched, the electrical conduction through this layer may be ignored $\left(G_{p 1}=0\right)$, and equation (4) can be rewritten as

$$
\frac{V}{\Delta V}=\frac{\alpha \pi}{\ln (2)} \frac{1}{F}
$$

It can be deduced that $V / \Delta V$ tends to $\alpha \pi / \ln 2$ when $F$ tends to 1 , and begins to increase towards infinity when $F$ tends to 0 . Since in the etched sample the transversal conductance $G_{\mathrm{t}}$ associated with the IB layer/Si substrate junction is perceived like a serial conductance with the conductance of the contacts, it does not affect the sheet resistance measurement obtained with the van der Pauw method. Nevertheless, from equation (5) we would obtain information on $G_{\mathrm{t}}$ from the experimental measurement of the $V / \Delta V$ relation.

In figure 3 the experimental behaviour observed for the $V / \Delta V$ relation of the etched sample is in agreement with the model prediction. However, even though there is no electrical conduction through the implanted layer $\left(G_{p 1}=0\right)$, a dependence with the decoupling function $F$ is observed, in agreement with equation (5). This is due to the presence of transversal electrical conduction $\left(G_{\mathrm{t}} \neq 0\right)$ through the junction between the Ti-implanted layer under the $\mathrm{Al}$ contacts and the unimplanted Si substrate (represented in the sketch of figure $2(a)$ ). Since $G_{\mathrm{t}}$ depends on the IB energy position, this transversal electrical conduction could be strong evidence of IB formation in Ti-implanted layer. The agreement in the trend between the experimental results and the analytical twolayer model justifies the use of a lumped parameter model to describe the physical phenomena.

The discrepancies observed in the $V / \Delta V$ relation between the theory and the experiment in the etched samples could be attributed to current leakages in the superficial states or other alternative current paths that can begin to be important when the junction is electrically blocked.

Finally, in figure 4 we show the electrical decoupling function $F$ obtained from equations (2) and (4) with the experimental measurements of the $V / \Delta V$ relation from the non-etched Ti-implanted sample and the function $F$ obtained from equation (5) with the experimental measurements of the $V / \Delta V$ relation from the etched Ti-implanted sample. We also obtained the function $F$ from the fitting of the experimental data of the non-etched sample with the analytical model [11]. As can be observed, in all the shown curves, the $F$ function varies from 1 to 0 gradually, as the temperature decreases, in agreement with the decoupling electrical effect observed in the junction between the implanted and unimplanted layer.

Differences in the $F$ function between the etched and non-etched samples are detected. Since the measurements of the sheet resistance and Hall effect are carried out 


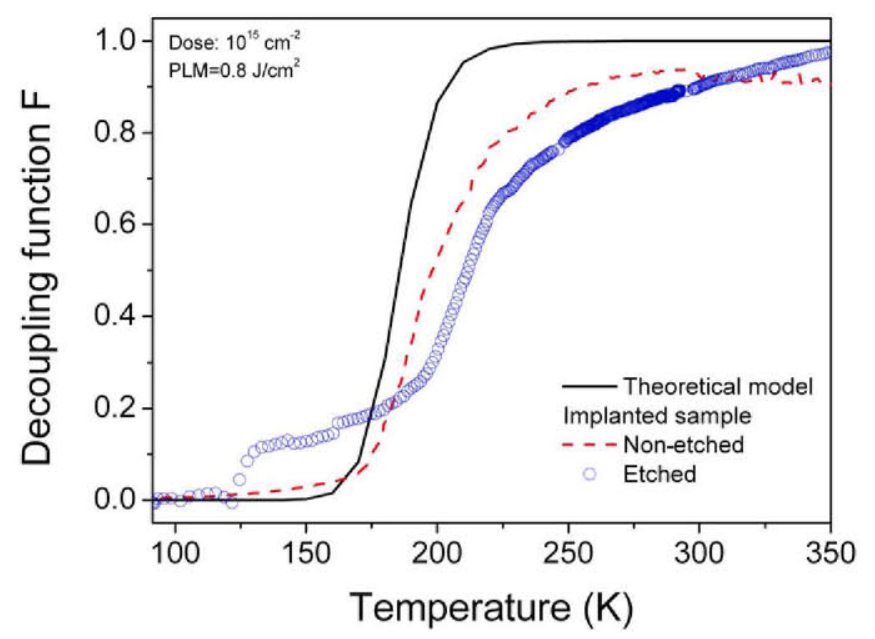

Figure 4. Electrical decoupling function $F$, obtained from the $V / \Delta V$ relation of the etched, non-etched and the fit to the two-layer model of the Ti-implanted silicon samples [11].

imposing a constant current between two contiguous contacts, if the implanted layer is etched, longitudinal conduction appears only along the Si unimplanted layer. It forces the measuring system to increase the voltage between the contacts to introduce the established measurement current. In consequence, a dependence on the input voltage of the electrical decoupling effect could be expected in this sample. This could indicate that the transversal resistance $R_{\mathrm{t}}$ could depend on the voltage applied to inject the measurement current and it could explain the differences observed in the electrical decoupling $F$ function obtained between the nonetched and the etched samples.

The differences observed between the experimental measurements and the theoretical predictions could be attributed to the model assumption of an abrupt junction between the Ti-supersaturated layer and the Si substrate, which is not completely achieved experimentally as can be appreciated from the Ti concentration depth profiles obtained after PLM processes [14]. In the case of the etched structure, additional current paths could begin to be appreciable, as was suggested in the $V / \Delta V$ function analysis.

\section{Conclusions}

We studied the current limitation effect responsible for electrical decoupling in a bilayer formed by a Ti-supersaturated $\mathrm{Si}$ layer where IB is formed on top of a $\mathrm{Si}$ substrate. In this work, we showed that the sheet resistance and Hall effect behaviour in the bilayers with the Ti-supersaturated $\mathrm{Si}$ layer, chemically etched but preserving the electrical contacts, returns to the unimplanted silicon substrate behaviour. It rules out the attribution of this anomalous behaviour to non-ohmic behaviour of the contacts with temperature and proves that the electrical bilayer decoupling produced at the junction between the Ti-supersaturated $\mathrm{Si}$ layer and the $\mathrm{Si}$ substrate is ultimately responsible for the anomalous rectifying effect observed in the electrical measurements. However, the electrical behaviour of the $V / \Delta V$ function of the etched sample is not the same as that expected from the unimplanted Si substrate. This is produced by the transversal current flow from the Ti-supersaturated Si layer to the Si substrate due to the bilayer junction remaining under the electrical contacts that has not been etched. However, we found that the analytical electrical model proposed to explain the observed electrical measurements in the IB materials formed in the bilayers can also explain the observed behaviour of the $V / \Delta V$ function in the new etched structure.

The electrical decoupling function $F$ obtained from the experimental measurements of the etched sample using the bilayer analytical model is compared with the same function obtained from the experimental results and theoretical calculations of the non-etched bilayer. Although some differences can be appreciated, all the results follow the same trend.

The experimental measurements performed in this work are in agreement with the previously reported analytical model that explains the electrical rectifying effect in a bilayer in terms of IB formation in a Ti-supersaturated Si layer, and constitutes further evidence of the formation of the IB.

\section{Acknowledgments}

The authors would like to thank the CAI de Técnicas Fisicas of the Universidad Complutense de Madrid for ion implantations, metallic evaporations and measurements with the optical profilometer. This work was partially supported by the Project NUMANCIA II (Grant No S-2009/ENE/1477) funded by the Comunidad de Madrid. Research by E GarcíaHemme has been partly supported by a PICATA predoctoral fellowship of the Moncloa Campus of International Excellence (UCM-UPM). DP and JO are grateful to Professors A Luque and A Martí for useful discussions and guidance and also acknowledge financial support from the MICINN within the program Juan de la Cierva (JCI-2011-10402 and JCI-201111471).

\section{References}

[1] Luque A and Martí A 1997 Phys. Rev. Lett. 785014

[2] Shockley W and Queisser H 1961 J. Appl. Phys. 32510

[3] Martí A, Antolín E, Stanley C R, Farmer C D, López N, Díaz P, Cánovas E, Linares P G and Luque A 2006 Phys. Rev. Lett. 97247701

[4] López N, Reichertz L A, Yu K M, Campman K and Waluckiewicz W 2011 Phys. Rev. Lett. 106028701

[5] Luque A, Martí A, Antolín E and Tablero C 2006 Physica B 382320

[6] Winkler M T, Recht D, Sher M J, Said A J, Mazur E and Aziz M J 2011 Phys. Rev. Lett. 106178701

[7] Yu K M, Walukiewicz W, Ager J W III, Bour D, Farshchi R, Doubon O D, Li S X, Sharp D and Haller E E $2006 \mathrm{Appl}$. Phys. Lett. 88092110

[8] Bob B P, Kohno A, Charnvanichborikarn S, Warrender J M, Umezu I, Tabbal M, Williams J S and Aziz M J 2010 J. Appl. Phys. 107123506

[9] Olea J, González-Díaz G, Pastor D and Mártil I 2009 J. Phys. D: Appl. Phys. 42085110

[10] González-Díaz G, Olea J, Mártil I, Pastor D, Martí A, Antolín E and Luque A 2009 Sol. Energy Mater. Sol. Cells 931668 
[11] Olea J, González-Díaz G, Pastor D, Mártil I, Antolín E, Martí A and Luque A 2011 J. Appl. Phys. 109063718

[12] Pastor D, Olea J, del Prado A, García-Hemme E, García-Hernansanz R and González-Díaz G 2012 Sol. Energy Mater. Sol. Cells 104 159-64

[13] Olea J, Pastor D, del Prado A, García-Hemme E, Mártil I and González Díaz G 2012 Thin Solid Films 5206614

[14] Olea J, Toledano-Luque M, Pastor D, San-Andrés E, Mártil I and González-Díaz G $2010 \mathrm{~J}$. Appl. Phys. 107103524
[15] Olea J, Toledano-Luque M, Pastor D, González-Díaz G and Mártil I 2008 J. Appl. Phys. 104016105

[16] Pastor D, Olea J, del Prado A, Mártil I, González-Díaz G, Ibáñez J, Cuscó R and Artús L 2011 Semicond. Sci. Technol. 26115003

[17] van der Pauw L J 1958 Phillips Research Reports 13 1-9

[18] Vossen J L and Kern W 1991 Thin film processes II (New York: Academic) p 438

[19] Sze S M and Kwok K Ng 2007 Physics of Semiconductor Devices 3rd edn (Hoboken, NJ: Wiley) pp 28-40 